\title{
Teaching Opioid Tapering Through Guided Instruction
}

Rebecca Claire Austin, PharmD, CPP; Carriedelle Wilson Fusco, NP; E. Blake Fagan, MD; Evan Drake, PharmD, CPP; Josh Pacious, MD; Hallum Dickens, MD; Shelley L. Galvin, MA; Courtenay Gilmore Wilson, PharmD, CDE, BCACP, BCPS, CPP

BACKGROUND AND OBJECTIVES: Given the current opioid epidemic, national practice guidelines and many state laws are shifting the treatment paradigm for chronic, noncancer pain to a judicious use of opioids. This has prompted a need to teach family medicine residents how to appropriately taper opioids. We created a multifaceted approach to integrate teaching of opioid tapering into a family medicine curriculum with an emphasis on guided instruction. We assessed the degree to which this curriculum affected guideline-concordant opioid prescribing within the family medicine practice.

METHODS: A retrospective chart review of 707 patients on chronic opioid therapy (COT) for noncancer pain was conducted before and after the incorporation of a guided instruction experience to the residency curriculum. The primary outcomes included the number of patients on chronic opioids, the average morphine equivalent daily (MED) per patient, the percentage of patients on $\geq 50$ MED or $\geq 90$ MED, and the number of patients on concomitant benzodiazepines.

RESULTS: Of the original 707 patients, 188 tapered off COT. Of those remaining on COT, the average MED did not change (53.4 \pm 76.9 vs $58.5+89.1$, $P=0.053)$. The percentage of patients on $\geq 50$ MED and $\geq 90$ MED decreased significantly $(30.6 \%$ vs $25.0 \%, P=0.001 ; 19.4 \%$ vs $14.0 \%, P=0.027)$. The total number of patients on concomitant benzodiazepine decreased from 212 to 131 .

CONCLUSIONS: Providing opportunities for guided instruction with opioid tapering allowed for an increased concordance with national practice guidelines.

(Fam Med. 2019;51(5):434-7.)

doi: 10.22454/FamMed.2019.502509

C hronic pain is a common problem that family physicians must manage. ${ }^{1}$ Few effective treatment modalities exist, which contributed to the overprescribing of opioids and the subsequent opioid epidemic. ${ }^{2,3}$ In 2016, the Centers for Disease Control and Prevention (CDC) released guidelines for the management of chronic pain. ${ }^{2}$ That same year, 46 governors signed $A$ Compact to Fight Opioid Addiction, focusing on reducing inappropriate prescribing. ${ }^{4}$ As of April 2018, 28 states have enacted opioid-limiting legislation. ${ }^{5}$

Several organizations describe opioid tapering techniques; however, the challenge often comes with patientspecific implementation. ${ }^{2,6-9}$ As the pain pendulum swings, residents must learn to taper opioids when appropriate. The average family medicine residency allocates 33 hours to pain education with topics ranging from assessment to therapies. ${ }^{10} \mathrm{In}$ 2017, the Association of American Medical Colleges highlighted efforts to increase opioid education. ${ }^{11} \mathrm{Re}$ cent studies demonstrated the use of skills-based and simulated learning to reinforce content taught via direct instruction. ${ }^{12-13}$ This study expands this by assessing the impact of an opioid tapering curriculum on the following primary endpoints: the number of patients on chronic opioid therapy (COT), average morphine equivalent daily (MED) per patient, percentage of patients on $\geq 50 \mathrm{MED}$ and $\geq 90 \mathrm{MED}$, and number of patients on concomitant benzodiazepines.

\section{Methods}

The University of North Carolina Health Sciences at the Mountain Area Health Education Center

From the University of North Carolina (UNC) Eshelman School of Pharmacy, Chapel Hill, NC (Drs Austin, Drake, and Wilson); Mountain Area Health Education Center (MAHEC), Asheville, NC (Drs Austin, Drake, Pacious, and Dickens); UNC Health Sciences at MAHEC (Drs Fagan and Wilson, and Mss Fusco and Galvin); and UNC School of Medicine, Chapel Hill, NC (Dr Wilson and Ms Galvin). 
is home to a family medicine residency with 34 residents, 20 faculty physicians, and a robust interdisciplinary care team treating approximately 23,000 patients.

\section{Baseline}

Following the release of the CDC guidelines, we completed a needs assessment to determine how well we adhered to the $12 \mathrm{CDC}$ recommendations. An EHR-generated report identified patients aged 18 years and older on COT, defined as three consecutive monthly opioid prescriptions. Cancer and palliative care patients were excluded. We found 707 (3.1\%) patients were on COT (Table 1). CDC recommendations 5 (prescribe lowest effective dose) and 11 (avoid concurrent benzodiazepine) were areas for most improvement. This needs assessment also prompted a naloxone coprescribing initiative (recommendation 8). Importantly, we began treating opioid use disorder in 2015 (recommendation 12).

\section{Intervention}

Subsequent to this needs assessment, in July 2016, we implemented a three-pronged opioid tapering curriculum: awareness, direct instruction, and guided instruction.

Awareness. All providers were given practice-wide and provider-specific data on the primary endpoints.

Direct Instruction. An annual, required 1-hour session was incorporated into the didactics and was repeated as a required faculty development seminar. Components of this session included a review of the CDC guidelines and role-playing scenarios. Tapering protocols based on national standards were reviewed in depth to ensure appropriate tapering. ${ }^{2,6}$

Guided Instruction. Within the longitudinal curriculum, each resident spent 4 half-days at minimum in the practice's interdisciplinary pain clinic and participated in one chronic pain group medical visit each year. As such, pain clinic and group were always staffed with at least one resident. During these experiences, residents had concentrated periods of time working with patients on COT in order to apply the skills learned in the direct instruction portion of the curriculum. This ensured that residents had ample practice with the tapering protocols.

\section{Evaluation}

A retrospective chart review in July 2017 examined the association of the intervention on the primary endpoints. Patients were considered active if they had an office visit in the past 12 months, and were considered no longer on COT if they have not received a prescription in the past 3 months. Pre- versus postintervention rate of $\mathrm{MED} \geq 50$ or $\geq 90$ was compared using McNemar's test; change in MED was compared using Wilcoxon signed-rank test. Binary logistic regression was conducted examining correlates of remaining an active patient and on COT. Our health-system's Institutional Review Board approved this project.

\section{Results}

Figure 1 describes the course of treatment for the baseline cohort of patients. The demographics of active patients is shown in Table 2 . The percentage of patients on $\geq 90$ and $\geq 50$ MED decreased significantly $(19.4 \%$ vs $14.0 \%, P=0.027 ; 30.6 \%$ vs $25.0 \%, P=0.001$, respectively). The

Table 1: Baseline Characteristics of Patients on Chronic Opioid Therapy $(n=707)$

\begin{tabular}{|l|c|}
\hline Age (years), M \pm SD & $62.5 \pm 15.2$ \\
\hline Female, $\mathrm{n}(\%)$ & $463(65.5)$ \\
\hline MED & \\
$\quad$ M \pm SD & $53.4 \pm 76.9$ \\
Median & 30 \\
$\quad$ min, max) & $(1.5-747.5)$ \\
\hline Benzodiazepine use, $\mathrm{n}(\%)$ & $212(30.0)$ \\
\hline
\end{tabular}

Abbreviation: MED, morphine equivalent daily.

Figure 1: Course of Treatment for Initial Cohort $(n=707)$

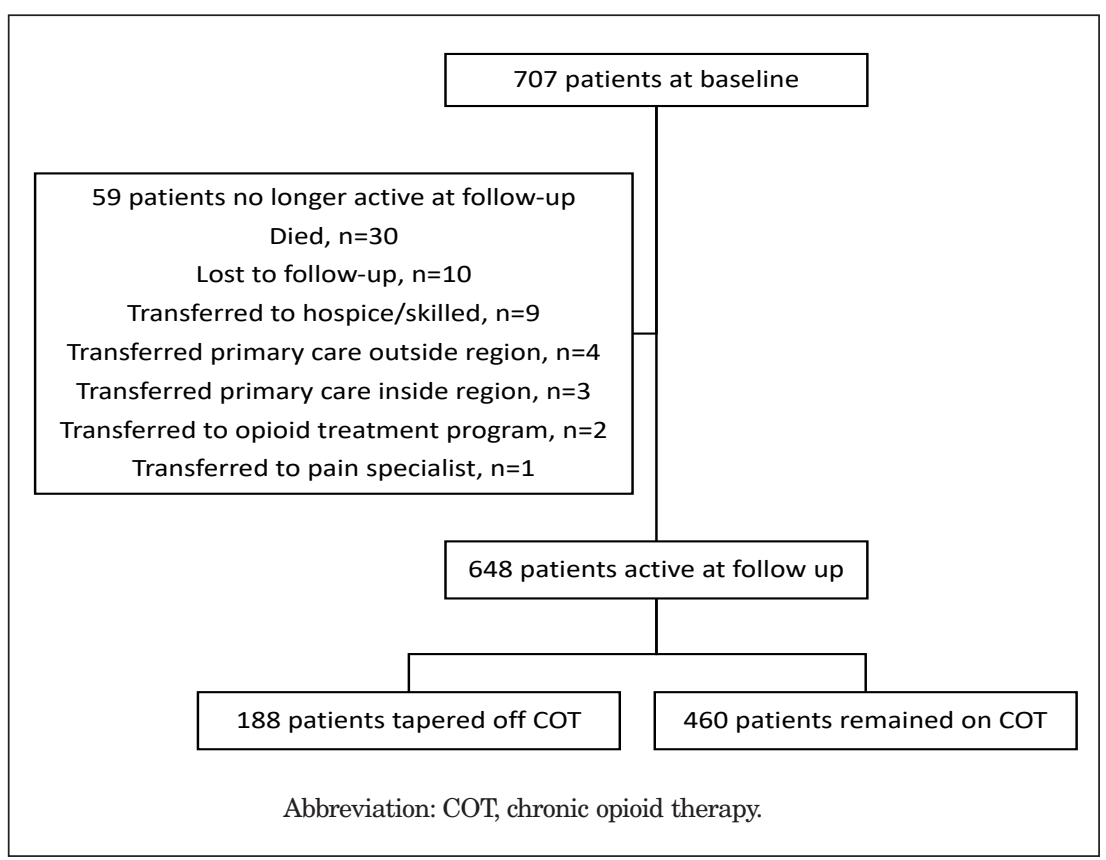


Table 2: Characteristics of Patients Active at Follow Up ( $n=648)$

\begin{tabular}{|l|c|}
\hline Age, $\mathrm{M} \pm \mathrm{SD}$ & $61.8 \pm 15.0$ \\
\hline Female, $\mathrm{n}(\%)$ & $425(65.6)$ \\
\hline Patients on chronic opioid therapy, n (\%) & $460(71.0)$ \\
\hline $\begin{array}{l}\text { MED } \\
\text { M } \pm \text { SD }\end{array}$ & $58.5 \pm 89.1$ \\
$\quad \begin{array}{l}\text { Median } \\
\text { (min, max) }\end{array}$ & $(0.83-840)$ \\
\hline Benzodiazepine use, $\mathrm{n}(\%)$ & $131(28.5)$ \\
\hline
\end{tabular}

Abbreviation: MED, morphine equivalent daily.

mean MED was not significantly different between the baseline cohort and patients who remained on COT $(53.4 \pm 76.9$ vs $58.5 \pm 89.1, P=0.053)$. The total number of patients on concomitant benzodiazepine decreased from 212 to 131 . Remaining an active patient was associated with younger age, and continuing COT was associated with higher baseline MED (Table 3).

\section{Discussion}

Through this intervention, we tapered $29 \%$ of patients off COT. Importantly, our retention rate remained over $90 \%$. For patients on $\mathrm{COT}$, there were higher rates of guideline-concordant care.

This opioid tapering curriculum utilized multiple learning modalities with the preponderance of time spent in guided instruction. While the practical aspects of opioid tapering may be taught through direct instruction, the art of opioid tapering requires a more active form of learning. Local trends mirror the results seen in this intervention. From 2016 to 2017, North Carolina decreased the number of opioids dispensed by $9.8 \%$, suggesting multiple statewide efforts to decrease opioid prescribing. ${ }^{14}$

The small scope of this study narrowed the endpoints specifically to the role of opioid tapering. Notably, this intervention occurred within a comprehensive approach to pain management including: counseling with behavioral medicine, maximizing nonopioid options, and ensuring access to nonpharmacological modalities. The emphasis and incorporation of these modalities in our care plan predates the educational intervention.

\section{Limitations}

The majority of patients on COT were assigned to faculty; however, the collaborative care model of pain clinic and group medical visits allowed residents direct experience with tapering opioids. We did not review the tapers employed to ensure fidelity to the protocols taught in the curriculum. We did not evaluate comorbid mental health diagnoses, which might affect patients' ability to discontinue opioids and contribute to benzodiazepine use.

\section{Conclusions}

The CDC guidelines set forth recommendations for the management of pain that have been broadly codified. We developed an opioid tapering curriculum with an emphasis on guided instruction that resulted in fewer patients on COT and more CDC guideline-concordant care. Further research is needed to assess the resident experience with this approach to teaching chronic pain management.

Table 3: Variables Associated With Remaining an Active Patient and on Chronic Opioid Therapy

\begin{tabular}{|c|c|c|c|c|c|c|}
\hline Baseline Characteristics & $\begin{array}{l}\text { Inactive at } \\
\text { Follow-up }\end{array}$ & Active at Follow-up & $\boldsymbol{P}$ & No COT at Follow-up & COT at Follow-up & $\boldsymbol{P}$ \\
\hline Patient, $\mathrm{n}(\%)$ & $59(8.3)$ & $648(91.7)$ & -- & $188(29.0)$ & $460(70.1)$ & -- \\
\hline $\mathrm{MED} \geq 50 \mathrm{mg} /$ day, $\mathrm{n}(\%)$ & $22(37.3)$ & $194(29.9)$ & $0.241^{*}$ & $23(12.2)$ & $171(37.2)$ & $<0.001^{*}$ \\
\hline Ages, $\mathrm{M} \pm \mathrm{SD}$ & $70.1 \pm 16.1$ & $61.8 \pm 15.0$ & $<0.001 \dagger$ & $60.9 \pm 16.5$ & $62.1 \pm 14.3$ & $0.083^{\dagger}$ \\
\hline Female, n $(\%)$ & $38(64.4)$ & $425(65.6)$ & $0.601^{\dagger}$ & $116(61.7)$ & $309(67.2)$ & $0.127^{\dagger}$ \\
\hline $\begin{array}{l}\text { MED } \\
\qquad \begin{array}{l}\text { M } \pm \text { SD } \\
\text { M } \\
\text { (min, max })\end{array}\end{array}$ & $\begin{array}{c}66.6 \pm 87.1 \\
37.5 \\
(5-450)\end{array}$ & $\begin{array}{c}52.2 \pm 75.9 \\
30 \\
(1.5-747.5)\end{array}$ & $0.054^{\dagger}$ & $\begin{array}{c}31.9 \pm 55.9 \\
15 \\
(1.5-480.0)\end{array}$ & $\begin{array}{c}60.5 \pm 81.3 \\
37.5 \\
(2.5-747.5)\end{array}$ & $<0.001^{\dagger}$ \\
\hline Benzodiazepine use, n(\%) & $19(32.2)$ & $193(29.8)$ & $0.461^{\dagger}$ & $51(27.1)$ & $142(30.9)$ & $0.763 \dagger$ \\
\hline $\begin{array}{l}\text { Provider, n(\%) } \\
\text { Faculty } \\
\text { Resident }\end{array}$ & $\begin{array}{c}52(8.5) \\
7(7.4)\end{array}$ & $\begin{array}{c}560(91.5) \\
88(92.6)\end{array}$ & $0.755^{\dagger}$ & $\begin{array}{c}168(30.0) \\
20(22.7)\end{array}$ & $\begin{array}{c}392(70.0) \\
68(77.3)\end{array}$ & $0.163 \dagger$ \\
\hline
\end{tabular}

Abbreviations: COT, chronic opioid therapy; MED: morphine equivalent daily.

$* \chi^{2}$ analysis

${ }^{\dagger}$ Binary logistic regression 
CORRESPONDING AUTHOR: Address correspondence to Dr Courtenay Gilmore Wilson, 123 Hendersonville Rd, Asheville, NC 28803. 828-257-4467. 828-257-4738. Courtenay.wilson@mahec.net.

\section{References}

1. Breuer B, Cruciani R, Portenoy RK. Pain management by primary care physicians, pain physicians, chiropractors, and acupuncturists: a national survey. South Med J. 2010;103(8):738747.

2. Dowell D, Haegerich TM, Chou R. CDC Guideline for prescribing opioids for chronic pain - United States, 2016. MMWR Recomm Rep. 2016;65(1):1-49

3. US Department of Health and Human Services (HHS), Office of the Surgeon General, Facing Addiction in America: The Surgeon General's Report on Alcohol, Drugs, and Health. Washington, DC: HHS, November 2016.

4. National Governors Association. A Compact to Fight Opioid Addiction. July 2016. http:// natlgovassoc.wpengine.com/news/a-compact-tofight-opioid-addiction/. Accessed July 31, 2018.

5. National Conference of State Legislatures. Prescribing Policies: States confront opioid overdose epidemic. http://www.ncsl.org/research/ health/prescribing-policies-states-confrontopioid-overdose-epidemic.aspx. Accessed July $31,2018$.
6. Washington State Agency Medical Directors Group. Interagency Guideline on Prescribing Opioids for Pain. http://www.agencymeddirectors.wa.gov/guidelines.asp. Accessed July 31 2018 .

7. US Department of Veterans Affairs. Opioid Taper Decision Tool. https://www.pbm.va.gov/ AcademicDetailingService/Documents/Pain Opioid_Taper_Tool_IB_10_939_P96820.pdf Accessed July 31, 2018.

8. Darnall BD, Ziadni MS, Stieg RL, Mackey IG, Kao MC, Flood P. Patient-centered prescription opioid tapering in community outpatients with chronic pain. JAMA Intern Med. 2018;178(5):707-708

9. Sullivan MD, Turner JA, DiLodovico C, D’Appollonio A, Stephens K, Chan YF. Prescription opioid taper support for outpatients wth chronic pain: a randomized controlled trial. J Pain. 2017;18(3):308-318

10. Zoberi K, Everard KM. Teaching chronic pain in the family medicine residency. Fam Med. 2018;50(1):22-27.

11. Association of American Medical Colleges. Academic Medicine's Response to Opioid Epidemic. Association of American Medical Colleges. https://aamc-black.global.ssl.fastly.net/ production/media/filer_public/75/fd/75fdc007d81d-4f4d-8e3a-e084ea9c344e/academic medicines_opioid_response_july_2017.pdf. Accessed December 7, 2018
12. Alford DP, Carney BL, Brett B, Parish SJ, Jackson AH. Improving residents' safe opioid prescribing for chronic pain using an objective structured clinical examination. J Grad Med Educ. 2016;8(3):390-397.

13. Kannampallil TG, McNutt R, Falck S, et al. Learning optimal opioid prescribing and monitoring: a simulation study of medical residents. JAMIA Open, Volume 1, Issue 2, 1 October 2018, Pages 246-254,

14. North Carolina Department of Health and Human Services. Opioid pills dispensed statewide are now decreasing. https://injuryfreenc. shinyapps.io/OpioidActionPlan/. Accessed December 14, 2018. 